\title{
Pharmacological inhibition of Eph receptors enhances glucose-stimulated insulin secretion from mouse and human pancreatic islets
}

\author{
R. Jain • D. Jain • Q. Liu • B. Bartosinska • J. Wang • \\ D. Schumann • S. G. Kauschke • P. Eickelmann • \\ L. Piemonti • N. S. Gray • E. Lammert
}

Received: 19 October 2012 / Accepted: 7 February 2013 /Published online: 9 March 2013

(C) Springer-Verlag Berlin Heidelberg 2013

\begin{abstract}
Aims/hypothesis Type 2 diabetes is characterised by impaired glucose-stimulated insulin secretion (GSIS) from pancreatic islets. Since erythropoietin-producing hepatoma (Eph)-ephrin bidirectional signalling fine-tunes GSIS from pancreatic beta cells, we investigated Eph receptor tyrosine kinases (RTK) as potential drug targets for selectively increasing GSIS.

Methods Insulin secretion assays were carried out using mouse and human pancreatic islets as well as mouse insulinoma (MIN6) cells in the presence or absence of two Eph RTK inhibitors. Furthermore, the most potent inhibitor was injected into mice to evaluate its effects on glucose tolerance and plasma insulin levels.
\end{abstract}

Electronic supplementary material The online version of this article (doi:10.1007/s00125-013-2877-1) contains peer-reviewed but unedited supplementary material, which is available to authorised users.

R. Jain $\cdot$ D. Jain $\cdot$ B. Bartosinska $\cdot$ E. Lammert $(\bowtie)$

Institute of Metabolic Physiology and German Diabetes Center,

Heinrich-Heine University Düsseldorf, Universitätsstrasse 1,

40225 Düsseldorf, Germany

e-mail: Lammert@uni-duesseldorf.de

Q. Liu $\cdot$ J. Wang $\cdot$ N. S. Gray

Biological Chemistry and Molecular Pharmacology, Dana Farber

Cancer Institute, Harvard Medical School, Boston, MA, USA

D. Schumann $\cdot$ S. G. Kauschke $\cdot$ P. Eickelmann

Cardiometabolic Diseases Research, Boehringer Ingelheim

Pharma GmbH \& Co. KG, Biberach an der Riß, Germany

L. Piemonti

Beta Cell Biology Unit, Diabetes Research Institute (HSR-DRI),

San Raffaele Scientific Institute, Milan, Italy

E. Lammert

Max Planck Institute of Molecular Cell Biology and Genetics,

Dresden, Germany
Results We showed that the Eph RTK inhibitors selectively increased GSIS from MIN6 cells as well as mouse and human islets. Our results also showed that the insulin secretory effects of these compounds required Eph-ephrin signalling. Finally, pharmacological inhibition of Eph receptor signalling improved glucose tolerance in mice.

Conclusions/interpretation We showed for the first time that Eph RTKs represent targets for small molecules to selectively increase GSIS and improve glucose tolerance.

Keywords Compound · Eph · Ephrin · Glucose tolerance · Insulin secretion $\cdot$ Pancreatic islets

$\begin{array}{ll}\text { Abbreviations } \\ \text { Eph } & \text { Erythropoietin-producing hepatoma } \\ \text { GLP-1 } & \text { Gucagon-like peptide-1 } \\ \text { GSIS } & \text { Glucose-stimulated insulin secretion } \\ \text { IM } & \text { Imatinib mesylate } \\ \text { NMR } & \text { Nuclear magnetic resonance } \\ \text { RTK } & \text { Receptor tyrosine kinases } \\ \text { WST-1 } & \text { Water-soluble-tetrazolium-1 }\end{array}$

\section{Introduction}

Insulin is secreted by pancreatic beta cells in response to elevated blood glucose levels. More specifically, glucose is taken up by the beta cells via glucose transporters (GLUT2 in rodents, GLUT1 and/or GLUT3 in humans), and its metabolism increases the ATP/ADP ratio [1-3]. The latter closes ATP-sensitive $\mathrm{K}^{+}$channels, resulting in depolarisation of the plasma membrane and subsequent opening of voltage-gated 
$\mathrm{Ca}^{2+}$ channels. The influx of $\mathrm{Ca}^{2+}$ finally triggers the exocytosis of insulin.

Physiological insulin secretion is also influenced by many other factors, for example cell-cell communication between the beta cells in the pancreatic islets [4]. Konstantinova et al previously showed that this cellular communication improves glucose-stimulated insulin secretion (GSIS) in part via erythropoietin-producing hepatoma (Eph)A forward and ephrin-A reverse signalling $[5,6]$. While EphA receptors belong to the family of receptor tyrosine kinases (RTKs), their ligands, the ephrin-As, are glycosylphosphatidylinositol (GPI)-linked cell surface proteins. Together they mediate EphA-ephrin-A bidirectional signalling [7-9]. Adult mouse pancreatic islets express several, partially redundant EphA receptors (EphA1, EphA2, EphA4, EphA5, EphA7 and EphA8), whereas only three, partially redundant ephrin-A ligands, ephrin-A1, ephrin-A5 and to a lesser extent ephrin-A2, are expressed [5].

Since the activation of EphA RTKs suppresses insulin secretion [5], we investigated whether pharmacological interference with EphA forward-signalling would improve GSIS from pancreatic islets. Imatinib mesylate (IM; Gleevec, Axon Medchem, Groningen, the Netherlands), a small-molecule RTK inhibitor currently in use for the treatment of some types of cancer [10], has recently been shown to have beneficial effects on blood glucose levels by increasing insulin sensitivity in peripheral organs [11]. This finding demonstrates that rather non-selective RTK inhibitors could have some, as yet to be explored, therapeutic potential for the treatment of diabetes.

Here, we provide a proof-of-concept study showing that small molecular weight compounds can target Eph receptors to selectively increase glucose-stimulated rather than basal insulin secretion. We previously showed that Eph RTKs are partially dephosphorylated at high glucose concentrations, and that the extent of their tyrosine phosphorylation is inversely related to the extent of GSIS from pancreatic islets (Fig. 1a). In this study, we now report that 'type II' pan-Eph RTK inhibitors can further dephosphorylate EphA RTKs at stimulatory glucose concentrations (Fig. 1b) and thus increase GSIS and improve glucose tolerance. Our findings therefore point to a new therapeutic avenue for the treatment of type 2 diabetes.

\section{Methods}

Compounds Compound 6 (see electronic supplementary material [ESM] Fig. 1a), compound 9 (ESM Fig. 1b) and controls were synthesised as previously described [12]. Based on ${ }^{1} \mathrm{H}$ nuclear magnetic resonance (NMR) spectroscopy, their purity was estimated to be $95-99 \%$ (ESM Figs 2 and 3). IM was purchased from Axon Medchem (Groningen, the Netherlands) and exendin-4 from Genscript USA (Piscataway, NJ, USA).
NMR spectroscopy Compound 6 and compound 9 were dissolved in ${ }^{2} \mathrm{H}$-labelled DMSO solution at a concentration of $10 \mathrm{mmol} / \mathrm{l}$ each. The spectrum was subsequently acquired using a $600 \mathrm{MHz}$ Varian NMR instrument (Agilent-Varian, Santa Clara, CA, USA).

Pharmacokinetic studies Pharmacokinetic studies of compounds 6 and 9 were carried out by Sai Advantium Pharma, Hyderabad, India, on 8- to 12-week-old, male Swiss albino mice at a concentration of $10 \mathrm{mg} / \mathrm{kg}$ body weight through the peritoneum. Blood was drawn at different time points, and compound concentrations were analysed by liquid chromatography (LC)-MS/MS.

Mouse model, pancreatic islets, glucose tolerance test, insulin tolerance test and measurement of plasma insulin $E f n a 5^{-/}$mice have previously been described $[5,13]$, and C57BL/6J mice were purchased from Janvier (Saint Berthevin, France). Mice were provided with standard laboratory chow and drinking water ad libitum. The local animal ethics committee approved all the experiments.

Glucose tolerance tests were performed on 8- to 12-weekold male $\mathrm{C} 57 \mathrm{BL} / 6 \mathrm{~J}$ mice by intraperitoneal injection into fasted mice of $2 \mathrm{~g}$ glucose $/ \mathrm{kg}$ body weight with or without $5 \mathrm{mg} / \mathrm{kg}$ body weight of compound 6 or $12 \mathrm{mg} / \mathrm{kg}$ body weight of compound 9. Insulin tolerance tests were performed on non-fasted, 8- to 12 -week-old male $\mathrm{C} 57 \mathrm{BL} / 6 \mathrm{~J}$ mice by intraperitoneal injection of $0.75 \mathrm{mU} / \mathrm{g}$ body weight human insulin (Berlinsulin H; Berlin-Chemie AG, Berlin, Germany). Plasma insulin levels were measured on 8- to 12-week-old male C57BL/6J mice or 10- to 14-month-old male Efna $5^{-/-}$ and control mice using an ultrasensitive rat insulin ELISA (Crystal Chem, Chicago, IL, USA). Male 8- to 12-week-old C57BL/6J mice or 6- to 8-month-old Efna $5^{-1-}$ and control mice were used to isolate pancreatic islets as previously described $[5,14]$. Human islets were isolated at the San Raffaele Scientific Institute from three different donors after ethical committee approval (ESM Table 1).

Cell culture MIN6 cells [15] were used from passage 27 to passage 35 and nucleofected (Lonza, Cologne, Germany) according to the manufacturer's instructions with either pEGFP or dominant-negative EphA5 plasmid.

Water-soluble tetrazolium-1 viability assay, insulin secretion and content Islet viability was estimated by water-soluble tetrazolium-1 (WST-1) assay (Roche Diagnostics, Penzberg, Germany) by static incubation at $37^{\circ} \mathrm{C}$ for 3-4 h. For insulin secretion measurements, islets and MIN6 cells were maintained at $37^{\circ} \mathrm{C}$ for $1 \mathrm{~h}$ in $2 \mathrm{mmol} / 1$ or $25 \mathrm{mmol} / \mathrm{l}$ glucose with or without $100 \mathrm{nmol} / \mathrm{l}$ compound 6 , compound 9 or IM. The amount of insulin secreted into the buffer along with the amount of total insulin in MIN6 or 
a
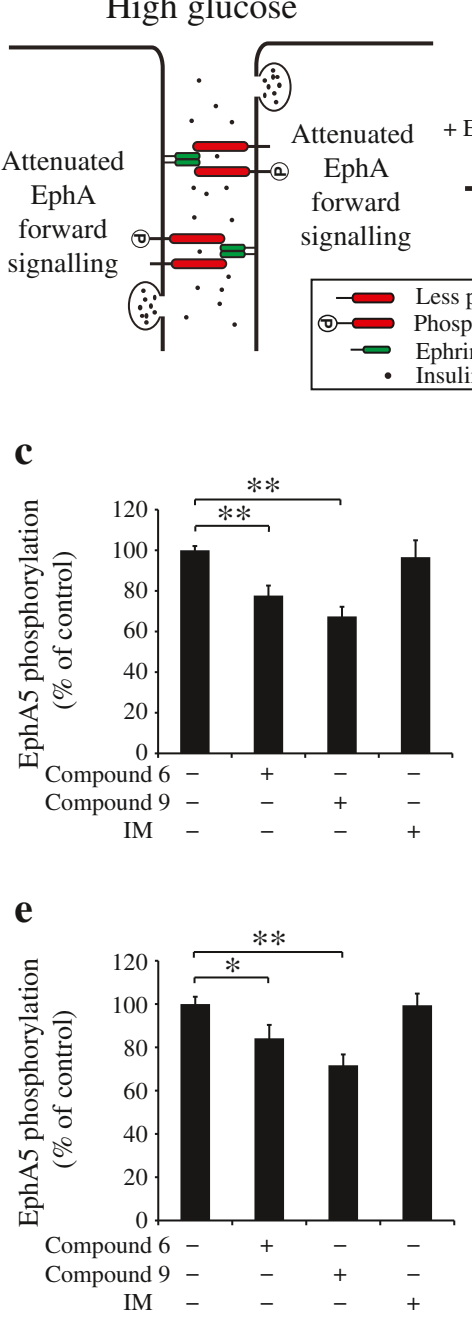

b High glucose

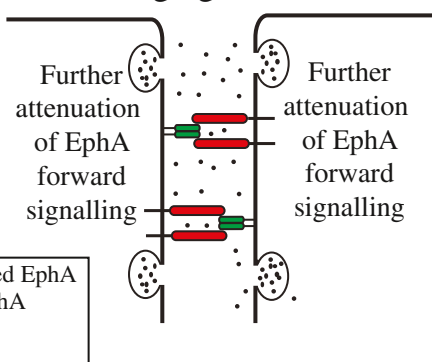

d

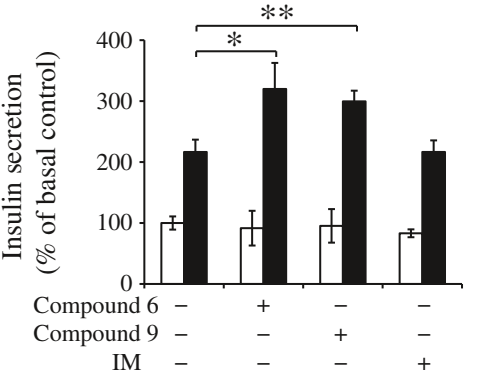

f

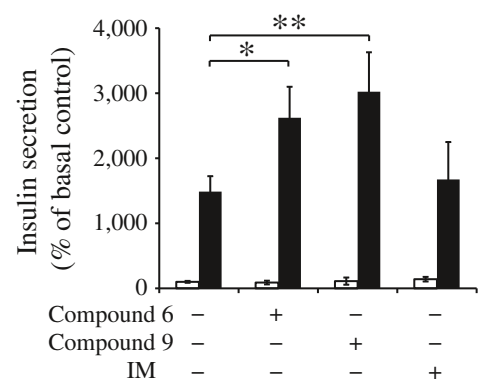

g

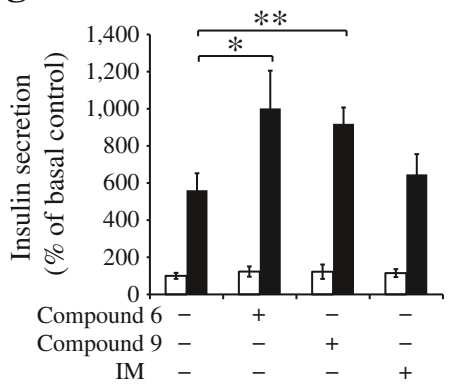

Fig. 1 Pharmacological inhibition of Eph RTK phosphorylation increases GSIS. $(\mathbf{a}, \mathbf{b})$ Model showing parts of two adjacent pancreatic beta cells that interact via Ephs (red; plain red symbol, less phosphorylated EphA; red symbol with encircled P, phosphorylated EphA) and ephrins (green). Eph receptors are partially dephosphorylated at high, stimulatory glucose concentrations (a). This dephosphorylation at high glucose concentrations, due to protein tyrosine phosphatase activity, attenuates EphA forward signalling, which inhibits the secretion of insulin (black dots). The aim of this study was to further dephosphorylate the Eph receptors using small-molecule Eph RTK inhibitors, thus further enhancing GSIS (b). (c, e) Mouse insulinoma cells (MIN6) (c) and mouse pancreatic islets (e) were treated with $100 \mathrm{nmol} / \mathrm{l} \mathrm{Eph}$ inhibitors, i.e. compound 6 and compound 9, and $100 \mathrm{nmol} / \mathrm{l}$ RTK inhibitor IM (Gleevec). The percentage of EphA5 phosphorylation was calculated after performing phospho-EphA5 and total EphA5 ELISA, and is shown in the presence or absence of the compounds. Phosphorylation levels were normalised to the amount of total EphA5 protein. (d, f, $\mathbf{g}$ ) Insulin secretion at $2 \mathrm{mmol} / \mathrm{l}$ glucose (white bars) and $25 \mathrm{mmol} / 1$ glucose (black bars). The secreted insulin was first normalised to the total insulin content and then adjusted to $100 \%$ basal control. The assay was carried out in the presence of compound 6, compound 9 and IM at a concentration of $100 \mathrm{nmol} / \mathrm{l} \mathrm{each}$. Both compound 6 and compound 9 significantly increased GSIS from MIN6 cells (d), mouse pancreatic islets (f) and human pancreatic islets (g), whereas IM had no effect. Human islets were from a 49-year-old male donor with a BMI of $27.7 \mathrm{~kg} / \mathrm{m}^{2} .{ }^{*} p<0.05, * * p<0.01$ (two-tailed Student's $t$ test). All values are means $\pm \mathrm{SD} ; n=3-4$ 
islet cell lysates was measured using an ultrasensitive rat insulin ELISA (Crystal Chem). Secreted insulin was first normalised to total insulin content. Subsequently, basal insulin secretion under control conditions was set to $100 \%$, and all other values were presented as a percentage of this. Raw data are shown in ESM Table 2.

Total EphA5 and phospho-EphA5 content Total EphA5 and phospho-EphA5 were measured in MIN6 cells and islets using an ELISA (R\&D Systems, Wiesbaden-Nordenstadt, Germany), according to the manufacturer's instructions. Phospho-EphA5 was normalised to the total EphA5 content and presented as a percentage of the DMSO control.

Statistical analysis A Student's $t$ test with a two-tailed distribution and two-sample unequal variance was used to determine statistical significance. In all tests, only two groups were analysed, which had only one changed variable. To further test the correctness of the statistical significance in Figs $2 \mathrm{e}$ and $3 \mathrm{c}$, two-way ANOVA followed by Bonferroni correction was carried out using GraphPad PRISM 5.0d (GraphPad, La Jolla, CA, USA; ESM Table 3).

\section{Results}

Pharmacological inhibition of Eph receptor phosphorylation increases GSIS We first investigated whether two small molecular weight Eph inhibitors, namely compound 6 and compound 9 (ESM Fig. 1a and 1b), could reduce the phosphorylation levels of EphA5 in MIN6 cells and mouse pancreatic islets at a high, stimulatory glucose concentration (Fig. 1c and e). EphA5 was chosen from the eight EphA receptors expressed in mouse islets, since it was shown to be produced in human islets and represents a well-characterised member of the EphA receptor family [5]. Treatment with the two compounds was found to lead to a small but significant dephosphorylation of EphA5 (Fig. 1c, e). In contrast, treatment with IM (ESM Fig. 1c), taken as a control RTK inhibitor with little affinity for Eph RTKs, did not inhibit EphA5 phosphorylation (Fig. 1c, e). In turn, IM reduced the phosphorylation of cKit, a non-Eph RTK (ESM Fig. 4), whereas compounds 6 and 9 had no effect on cKit phosphorylation.

Next, we treated MIN6 cells, mouse islets and human islets with these compounds. Importantly, both Eph RTK inhibitors significantly and selectively increased GSIS (Fig. 1d, f, g), whereas IM had no effect. Since islet preparations can differ, human islets were isolated from three different donors and subsequently analysed (Fig. 1g, ESM Fig. 5). Importantly, both compounds significantly increased GSIS from all three preparations of human islets. Furthermore, two compounds, ALW-II-41-26 (ESM Fig. 6a) and

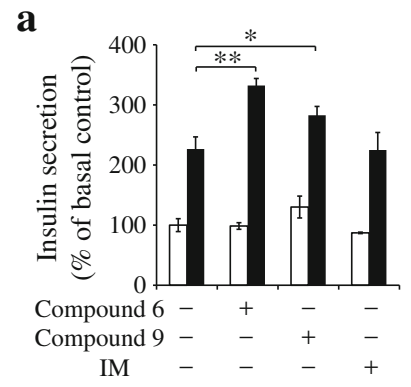

b
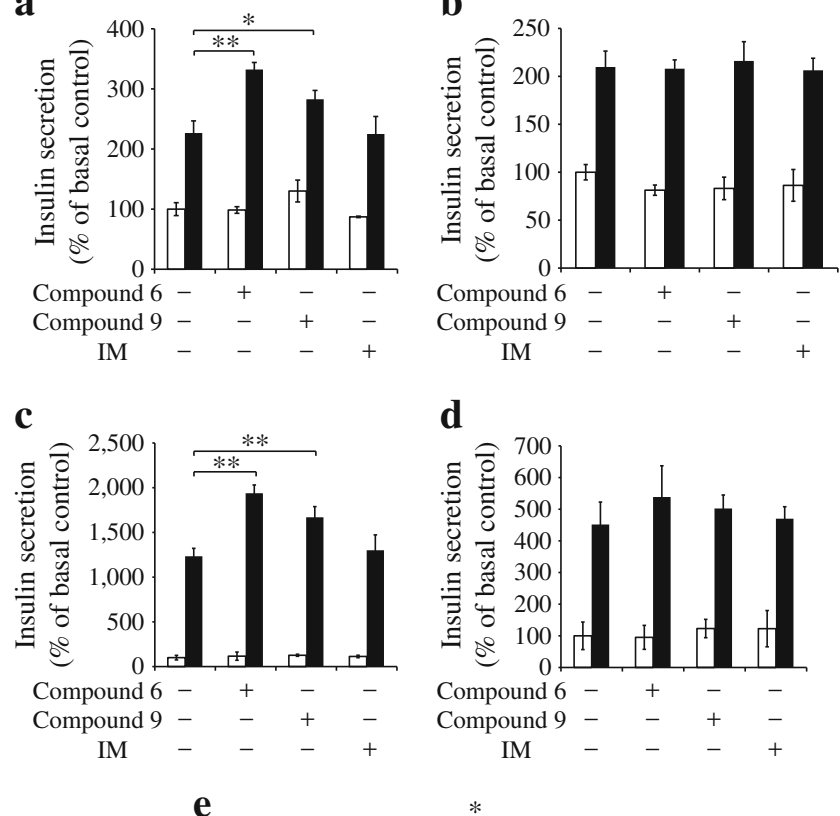

d
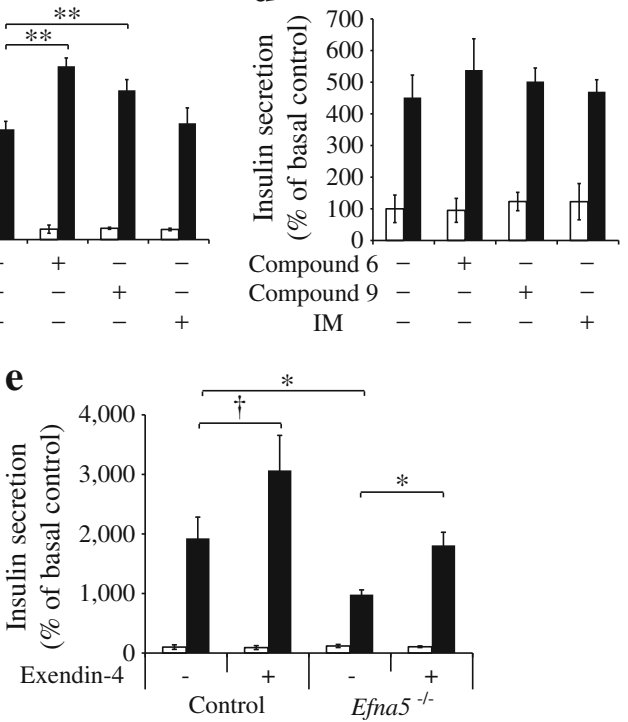

Fig. 2 Mechanism by which Eph RTK inhibitors increase GSIS. (a, b) Insulin secretion assays using MIN6 cells transfected with either an empty expression vector (a) or a vector expressing a dominant-negative-EphA5 lacking the cytoplasmic signalling domain (b). Cells were treated with $100 \mathrm{nmol} / \mathrm{l}$ compound 6, compound 9 or IM. (c, d) Insulin secretion assays using pancreatic islets isolated from control littermates (c) or Efna $5^{-/-}$mice (d). Islets were treated with $100 \mathrm{nmol} / \mathrm{l}$ compound 6, compound 9 or IM. (e) Insulin secretion assays using control and ephrinA5-deficient islets in the absence $(-)$ or presence $(+)$ of $0.1 \mathrm{nmol} / 1$ exendin-4, a GLP-1 receptor agonist. White bars, insulin secreted at $2 \mathrm{mmol} / 1$ glucose; black bars, insulin secreted at $25 \mathrm{mmol} / 1$ glucose from pancreatic islets or MIN6 cells. ${ }^{*} p<0.05,{ }^{*} p<0.01,{ }^{\dagger} p=0.057$ (twotailed Student's $t$ test). All values are means \pm SD; $n=3$

ALW-II-50-1 (ESM Fig. 6b) were chosen as controls, as they had structures similar to compound 6 and compound 9 , respectively, but had no significant activity towards EphA5 (data not shown). Neither of these control compounds increased GSIS from mouse islets (ESM Fig. 6c). Finally, we tested the toxicity of compounds 6 and 9 using a WST-1 mitochondrial function assay. Neither compound inhibited the mitochondrial function of mouse and human islets, indicating that they did not negatively affect islet viability (ESM Fig. 7).

Mechanism of Eph RTK inhibitors To further confirm that the two compounds did not increase GSIS by inhibiting RTKs other than the Eph receptors, we transfected MIN6 
a
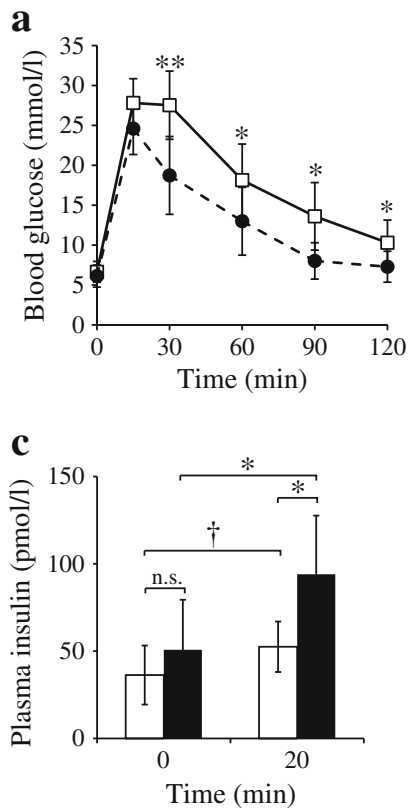

d
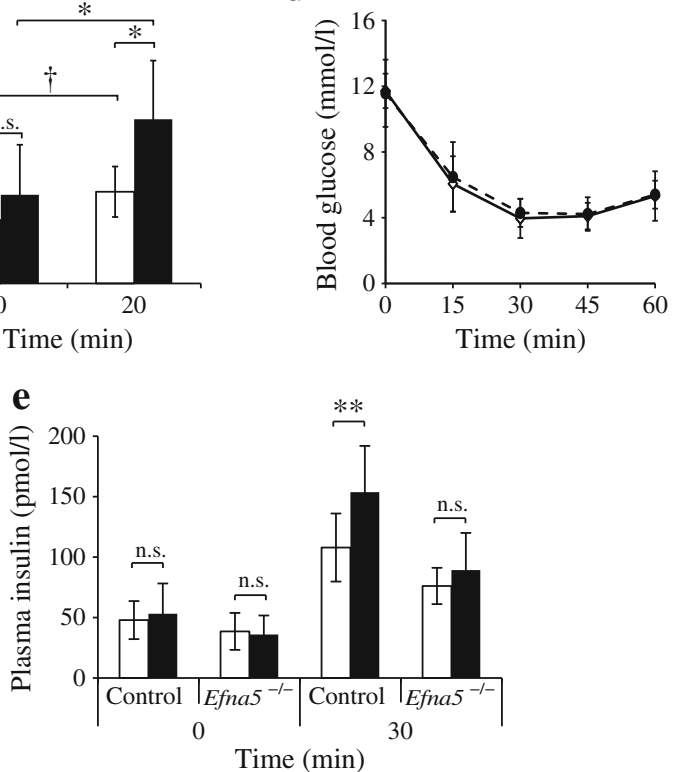

Fig. 3 Eph RTK inhibitor compound 6 improves glucose tolerance in mice. (a, b) Glucose tolerance tests (a) and AUC ( $\mathrm{mmol} / \mathrm{l} \times \mathrm{min})(\mathbf{b})$ of male $\mathrm{C} 57 \mathrm{BL} / 6 \mathrm{~J}$ mice after intraperitoneal administration of glucose ( $2 \mathrm{~g} / \mathrm{kg}$ body weight) together with $5 \mathrm{mg} / \mathrm{kg}$ body weight of compound 6 (black circles in a) or vehicle (white squares in a). $n=7-8$. (c) Plasma insulin levels in male $\mathrm{C} 57 \mathrm{BL} / 6 \mathrm{~J}$ mice were measured before $(0 \mathrm{~min})$ and $20 \mathrm{~min}$ after intraperitoneal administration of glucose $(2 \mathrm{~g} / \mathrm{kg}$ body weight) along with $5 \mathrm{mg} / \mathrm{kg}$ body weight of compound 6 (black bars) or vehicle (white bars). $n=7-8$. (d) Insulin tolerance tests were performed on male $\mathrm{C} 57 \mathrm{BL} / 6 \mathrm{~J}$ mice using an intraperitoneal injection of $0.75 \mathrm{U} / \mathrm{kg}$ body weight regular human insulin, along with $5 \mathrm{mg} / \mathrm{kg}$ body weight of compound 6 (black circles) or vehicle (white squares). $n=8$. (e) Plasma insulin levels in male control and $E f n a 5^{-1}$ mice were measured before $(0 \mathrm{~min})$ and $30 \mathrm{~min}$ after intraperitoneal administration of glucose $(2 \mathrm{~g} / \mathrm{kg}$ body weight) along with $5 \mathrm{mg} / \mathrm{kg}$ body weight of compound 6 (black bars) or vehicle (white bars). $n=7-10 .{ }^{* *} p<$ $0.01,{ }^{*} p<0.05,{ }^{\dagger} p=0.079$ (two-tailed Student's $t$ test). All values are means \pm SD

cells with a dominant-negative form of EphA5, which lacked an RTK domain. MIN6 cells transfected with the control vector were found to have increased GSIS upon compound treatment (Fig. 2a), whereas cells expressing the dominantnegative EphA5 showed no response (Fig. 2b).

We then asked whether an interaction of the EphA receptors with ephrin-A5, one of the two ephrin-A ligands most strongly expressed in beta cells, was required for the compounds to have an effect. Thus, ephrin-A5-deficient mouse islets were treated and GSIS was measured. Islets from control littermates producing ephrin-A5 showed a normal increase in GSIS upon compound treatment (Fig. 2c). In contrast, the compounds failed to significantly increase GSIS in ephrin-A5-deficient islets (Fig. 2d). To rule out the possibility that the lack of ephrin-A5 generally excluded a pharmacologically driven increase in GSIS, we treated ephrin-A5 deficient islets with the glucagon-like peptide-1 (GLP-1) analogue exendin-4, a drug in clinical use since 2005. Importantly, exendin-4 increased GSIS from both control and ephrin-A5-deficient islets (Fig. 2e). In sum, the data suggest that compounds 6 and 9 selectively increased GSIS by inhibiting Eph receptor tyrosine phosphorylation.

Eph RTK inhibition improves glucose tolerance in mice Finally, we asked whether the compounds would also increase plasma insulin content and glucose tolerance in vivo. Compound 6 was found to reach a more than 20 -fold higher plasma concentration compared with compound 9 (ESM Fig. 8), and corresponding to the better pharmacokinetics, compound 6 more significantly improved glucose tolerance in mice compared with the other compound (compare Fig. 3a, b and ESM Fig. 9). In addition, compound 6 significantly enhanced plasma insulin levels $20 \mathrm{~min}$ after glucose injection compared with controls (Fig. 3c), whereas insulin sensitivity remained unchanged (Fig. 3d). Finally, treatment of $E$ fna $5^{-/-}$mice with compound 6 failed to significantly increase plasma insulin levels (Fig. 3e). We conclude that pharmacological inhibition of Eph receptors with small molecules enhances glucose-induced plasma insulin levels and improves glucose tolerance in vivo.

\section{Discussion}

The present study shows that Eph RTKs can serve as targets for small molecules in order to specifically enhance GSIS from mouse and human pancreatic islets. In addition, the data reveal that small molecular weight Eph inhibitors can increase glucose-induced plasma insulin levels and improve glucose tolerance in mice. Both Eph RTK inhibitors and their control compounds, including IM, are type II kinase inhibitors [12, 16]. As there are no reported Eph inhibitors that are selective for a single Eph RTK family member, these compounds are pan-Eph inhibitors, although they exhibit substantially reduced affinity for EphA1, EphA6, EphA7 and EphB1 [12]. Importantly, IM and the two control compounds do not affect EphA5 receptor phosphorylation and consistently do not affect GSIS from both mouse and human islets. Conversely, in contrast to IM, neither Eph RTK inhibitor affects cKit phosphorylation, but they increase GSIS. 
The two Eph RTK inhibitors (compounds 6 and 9) used in this study dephosphorylate EphA5 by $20-35 \%$ at high glucose concentrations. This moderate degree of inhibition can be explained by our previous finding that Eph receptors in pancreatic beta cells already have a low degree of phosphorylation at high glucose concentrations [5] and that Eph receptors - like many RTKs - are also phosphorylated by non-receptor tyrosine kinases, such as Src family kinases, which are not affected by Eph RTK inhibition [8]. Nevertheless, our genetic experiments using ephrin-A5-deficient mouse islets and $E f n a 5^{-/}$mice along with dominantnegative Eph receptor production in MIN6 cells all reveal that the small-molecule Eph RTK inhibitors (compounds 6 and 9) selectively increase GSIS via their effects on Ephephrin bidirectional signalling. This is because the cytoplasmic signalling domain of EphA5 and the Eph receptor ligand ephrin-A5 are both required in beta cells to observe the insulin-secreting effects of the two compounds.

Our study warrants investigations of small molecular weight Eph RTK inhibitors in order to develop new drugs for the treatment of type 2 diabetes. First, the compounds tested are not expected to introduce severe hypoglycaemia as a life-threatening adverse effect since they selectively increase GSIS rather than basal insulin secretion. Second, Eph-ephrin bidirectional signalling takes place in cells and tissues affected by long-term complications of diabetes, including the heart, blood vessels and neurones, and recent reports indicate that manipulation of Eph-ephrin signalling pathways can protect against renal, neuronal and cardiovascular pathologies [17-20]. Thus, further studies need to be performed to analyse whether small molecular weight Eph RTK inhibitors not only improve blood glucose control, as shown here, but can also protect against some of the longterm complications of diabetes, in particular those affecting the cardiovascular and nervous systems of diabetic patients.

Acknowledgements We thank all our colleagues in Düsseldorf for helpful advice and discussions, in particular A. Welters and J. Eglinger.

Funding R. Jain and E. Lammert were supported by CRC 590. E. Lammert and D. Jain were supported by the German Center for Diabetes Research (DZD e.V.) of the Federal Ministry for Education and Research (BMBF).

Duality of interest The authors declare that there is no duality of interest associated with this manuscript.

Contribution statement All authors contributed to study design, data collection, analysis and interpretation, reviewing or revising the manuscript, and approving the final draft for publication.

\section{References}

1. Rorsman P, Braun M (2013) Regulation of insulin secretion in human pancreatic islets. Annu Rev Physiol 75:2.1-2.25

2. Maedler K, Størling J, Sturis J et al (2004) Glucose- and interleukin-1beta-induced beta-cell apoptosis requires $\mathrm{Ca}^{2+}$ influx and extracellular signal-regulated kinase (ERK) $1 / 2$ activation and is prevented by a sulfonylurea receptor 1 /inwardly rectifying $\mathrm{K}^{+}$ channel 6.2 (SUR/Kir6.2) selective potassium channel opener in human islets. Diabetes 53:1706-1713

3. Kulkarni RN (2004) The islet beta-cell. Int J Biochem Cell Biol $36: 365-371$

4. Jaques F, Jousset H, Tomas A et al (2008) Dual effect of cell-cell contact disruption on cytosolic calcium and insulin secretion. Endocrinology 149:2494-2505

5. Konstantinova I, Nikolova G, Ohara-Imaizumi M et al (2007) EphA-ephrin-A-mediated beta cell communication regulates insulin secretion from pancreatic islets. Cell 129:359-370

6. Kulkarni RN, Kahn CR (2007) Ephs and ephrins keep pancreatic beta cells connected. Cell 129:241-243

7. Klein R (2009) Bidirectional modulation of synaptic functions by Eph/ephrin signaling. Nat Neurosci 12:15-20

8. Pasquale EB (2008) Eph-ephrin bidirectional signaling in physiology and disease. Cell 133:38-52

9. Pitulescu ME, Adams RH (2010) Eph/ephrin molecules-a hub for signaling and endocytosis. Genes Dev 24:2480-2492

10. Thanopoulou E, Judson I (2012) The safety profile of imatinib in CML and GIST: long-term considerations. Arch Toxicol 86:1-12

11. Han MS, Chung KW, Cheon HG et al (2009) Imatinib mesylate reduces endoplasmic reticulum stress and induces remission of diabetes in $\mathrm{db} / \mathrm{db}$ mice. Diabetes 58:329-336

12. Choi Y, Syeda F, Walker JR et al (2009) Discovery and structural analysis of Eph receptor tyrosine kinase inhibitors. Bioorg Med Chem Lett 19:4467-4470

13. Knoll B, Zarbalis K, Wurst W, Drescher U (2001) A role for the EphA family in the topographic targeting of vomeronasal axons. Development 128:895-906

14. Yesil P, Michel M, Chwalek K et al (2009) A new collagenase blend increases the number of islets isolated from mouse pancreas. Islets 1:185-190

15. Miyazaki J, Araki K, Yamato E et al (1990) Establishment of a pancreatic beta cell line that retains glucose-inducible insulin secretion: special reference to expression of glucose transporter isoforms. Endocrinology 127:126-132

16. Liu Y, Gray NS (2006) Rational design of inhibitors that bind to inactive kinase conformations. Nat Chem Biol 2:358-364

17. Baldwin C, Chen ZW, Bedirian A et al (2006) Upregulation of EphA2 during in vivo and in vitro renal ischemia-reperfusion injury: role of Src kinases. Am J Physiol Ren Physiol 291:F960 F971

18. Broqueres-You D, Lere-Dean C, Merkulova-Rainon T et al (2012) Ephrin-B2-activated peripheral blood mononuclear cells from diabetic patients restore diabetes-induced impairment of postischemic neovascularization. Diabetes 61:2621-2632

19. Dries JL, Kent SD, Virag JA (2011) Intramyocardial administration of chimeric ephrinA1-Fc promotes tissue salvage following myocardial infarction in mice. J Physiol 589:1725-1740

20. Li J, Liu N, Wang Y et al (2012) Inhibition of EphA4 signaling after ischemia-reperfusion reduces apoptosis of CA1 pyramidal neurons. Neurosci Lett 518:92-95 